\title{
Fixed Target Serial Data Collection at Diamond Light Source
}

Sam Horrell ${ }^{1}$, Danny Axford ${ }^{1}$, Nicholas E. Devenish ${ }^{1}$, Ali Ebrahim ${ }^{1}$, Michael A. Hough ${ }^{2}$, Darren A. Sherrell ${ }^{1,3}$, Selina L. S. Storm $^{1,4}$, Ivo Tews ${ }^{5}$, Jonathan A. R. Worrall ${ }^{2}$, Robin L. Owen ${ }^{1}$

${ }^{1}$ Diamond Light Source, Harwell Science and Innovation Campus ${ }^{2}$ School of Life Sciences, University of Essex ${ }^{3}$ X-ray Science Division, Argonne National Laboratory ${ }^{4}$ European Molecular Biology Laboratory, Hamburg Outstation c/o DESY ${ }^{5}$ Biological Sciences, Institute for Life Sciences, University of Southampton

\section{Corresponding Author}

Robin L. Owen

robin.owen@diamond.ac.uk

\section{Citation}

Horrell, S., Axford, D., Devenish, N.E.,

Ebrahim, A., Hough, M.A., Sherrell, D.A.,

Storm, S.L.S., Tews, I., Worrall, J.A.R.,

Owen, R.L. Fixed Target Serial

Data Collection at Diamond Light

Source. J. Vis. Exp. (168), e62200,

doi:10.3791/62200 (2021).

\section{Date Published}

February 26, 2021

DOI

$10.3791 / 62200$

URL

jove.com/video/62200

\section{Introduction}

Serial synchrotron crystallography (SSX) is an emerging method of data collection which was inspired by X-ray free electron lasers $(X F E L)^{1,2,3}$. At an XFEL, a single diffraction pattern is recorded from a usually very small protein crystal, before the crystal is destroyed by the extremely bright $X$ ray pulse. This means, typically, that a new crystal must be introduced into the X-ray beam to obtain another diffraction

\section{Abstract}

Serial data collection is a relatively new technique for synchrotron users. A user manual for fixed target data collection at I24, Diamond Light Source is presented with detailed step-by-step instructions, figures, and videos for smooth data collection. pattern ${ }^{4}$. This need to continually replenish crystals has driven the development of many serial sample delivery techniques ${ }^{5}$.

At synchrotrons, classic (non-serial) rotation crystallography methods are widely applied, exploiting a single large crystal which is rotated in an X-ray beam using a goniometer to collect a complete dataset for structure solution ${ }^{6}$. In order to 
increase the lifetime of crystals so that a complete dataset can be collected ${ }^{7,8}$, and also to facilitate shipping and automated sample transfer, crystals are cryocooled to $\sim 100 \mathrm{~K}$ for data collection. At intense microfocus beamlines, multi-crystal strategies are frequently employed as radiation damage can prohibit the collection of a complete dataset from a single crystal $\left.\right|^{9}, 10,11$. Despite the limits imposed by radiation damage, the number of crystals used remains relatively modest and the approach used is essentially identical to the single crystal experiment.

SSX, on the other hand, uses serial sample delivery to obtain single still diffraction patterns from thousands of randomly orientated crystals to generate a complete dataset. It is noted that serial techniques incorporating crystal rotation are under development ${ }^{12,13}$ though we focus on still, zero rotation, approaches. There are a wide variety of sample delivery systems with different advantages and disadvantages ${ }^{14}$, ranging from delivering a stream of crystals in a flow focused/ viscous jet ${ }^{15,16,17}$, microfluidic chip ${ }^{18,19}$, or crystals on a fixed target such as an etched silicon chip 20,21 . Typically, crystals are held at room temperature, allowing greater conformational diversity to be observed and providing a more physiologically relevant environment ${ }^{22}$. SSX enables the collection of very low dose datasets ${ }^{23}$, as the total dose of the dataset is equivalent to a single short X-ray exposure of one crystal. Another major advantage SSX provides is the study of protein dynamics through time-resolved methods, with reactions triggered by exposure to laser light $24,25,26,27$ or by mixing of crystals and ligand/substrate 28,29 . Using smaller crystals means laser light can penetrate the entirety of the crystal, uniformly initiating the reaction without multiphoton absorption to provide well defined reaction intermediates for diffraction data taken at different time points ${ }^{27}$. Use of larger crystals and rotation-based data collection methods suffers from a limited laser penetration depth, nonuniform or multiphoton activation, radiation damage, and mechanical overhead time within data sweeps, resulting in a mix of reaction intermediates that can prove difficult or impossible to interpret at faster reaction speeds. Smaller crystals provide a similar advantage in mixing experiments, as ligands can rapidly and more uniformly diffuse throughout the crystal, again allowing defined reaction intermediates to be recorded at different time delays $30,31,32$.

At Diamond's microfocus beamline 124 both conventional rotation and SSX experiments can be performed. Here a comprehensive protocol for SSX sample preparation and data collection using fixed targets at 124 and protocols for data analysis of serial data at Diamond are presented. While the manuscript and accompanying videos should allow users to carry out a successful SSX experiment at 124, it should be noted that this is a rapidly developing field and approaches are continually evolving. It should also be noted that serial methods are available at other synchrotron sources, including but not limited to Petra III (P14-TREXX), MAX IV (BioMAX) ${ }^{33}$, SLS (PXI and PXII) ${ }^{34}$, and NSLS (FMX) ${ }^{35}$. While the the specifics of serial data collection and processing will differ between sources, the core principles will remain the same. The protocols below should be seen to represent a starting point and a pathway to base camp rather than the summit of what might be achieved.

This protocol assumes the users have a protein or small molecule crystal system, from which a microcrystal slurry on the order of $0.5-2.0 \mathrm{~mL}$ with a good density of microcrystals per $\mathrm{mL}$ has been produced. Protocols for obtaining crystal slurries have been described previously ${ }^{36}$. Many different types of fixed target are available, the most commonly used at I24 utilize a precisely defined silicon chip. In order 
to differentiate from other chip layouts, below and in the beamline interface this is referred to as an 'Oxford chip'. As previously described the Oxford chip layout comprises $8 \times 8$ 'city blocks', each containing $20 \times 20$ apertures for a total of 25,600 apertures $^{20,21}$.

\section{Protocol}

\section{Preparing and loading a chip}

NOTE: The process occurs within a humidity-controlled environment (Figure 1), typically between $80 \%$ and $90 \%$ or higher relative humidity, to prevent protein crystals from drying out. Once loaded and sealed, crystals can survive for upwards of 24 hours. However, this is can vary greatly between crystal systems. Within the chamber a low powered vacuum pump attached to a loading stage to hold a silicon chip (Figure 1), a silicon chip, a chip holder with polyester foil (Figure 2), a p200 pipette, $200 \mu \mathrm{L}$ pipette tips, tweezers, filter paper and the protein crystal slurry are required.

1. Prepare a chip holder.

1. Cut two sheets of polyester foil into squares approximately $6 \mathrm{~cm} \times 6 \mathrm{~cm}$.

2. Lay the polyester sheets over the two base plates (large and small).

3. Fix the polyester sheets in place using the metal sealing rings.

4. Carefully pull on the excess polyester foil to remove any creases to make visualizing and centering samples easier later.

2. Select a silicon chip with appropriately sized apertures (7-30 $\mu \mathrm{m})$ relative to the size of the crystals.
3. Glow discharge the chip for 25 seconds at $0.39 \mathrm{mBar}$ and using a current of $15 \mathrm{~mA}$ to enable easy spreading of micro crystals on the chip.

4. Place the silicon chip on the chip loading stage using tweezers with the raised bars facing down.

5. Apply $200 \mu \mathrm{L}$ of the micro-crystal slurry to the flat side of the chip using a pipette.

6. Spread out the crystal slurry to cover all the "city-blocks" of the chip.

7. If the chip is damaged, cover any holes with a small piece of polyester foil or filter pipette tip to ensure an even vacuum can be applied.

8. Apply a gentle vacuum until all excess liquid has been sucked through the chip.

9. Remove the chip from the chip loading stage with tweezers.

10. Carefully blot the underside of the chip with filter paper to remove excess liquid.

11. Place the loaded chip on the larger half of the chip holder between the guide marks flat side down.

12. Seal the chip by placing the small half of the chip holder on top.

1. The two halves of the chip holder will snap into place. If the second half does not sit flush, spin the holder $180^{\circ}$ to properly align the magnets.

13. Screw the chip holder closed with hex bolts to fix the chip securely in place.

NOTE: Alternatively, a "chipless" chip can be loaded in a similar fashion, with a smaller volume of crystal slurry $(\sim 15 \mu \mathrm{L})$ sandwiched between the two layers of polyester foil in the chip holder ${ }^{37}$, or a smaller 
volume can be loaded using a $50 \mu \mathrm{m}$ thick double-sided adhesive spacer applied directly to the polyester foil as described previously ${ }^{38}$. The use of adhesive spacers also allows multiple samples (or variants of samples such as ligand soaks) to be loaded on each chipless chip. A complementary loading approach exploiting acoustic drop ejection (ADE) to load silicon chips can also be used at Diamond ${ }^{39}$. ADE allows chips to be loaded using smaller volumes of crystal slurry than pipette loading. It is a particularly useful technique when samples are scarce, though the chemical composition and viscosity of the slurry must be taken into consideration.

\section{GUI and setup at the beamline}

1. Perform all chip alignment and setup for data collection through a simple EPICS Display Manager (edm) graphical user interface (GUI) (Figure 3a). This provides a point-and-click interface to beamline instrumentation and provides input parameters for Python-based data collection. Sub windows provide additional control for collecting from sub regions of a sample holder (Figure 3b) or laser/LED pump-probe experiments (Figure 3c).

\section{Aligning the chip}

1. Place the loaded chip on the $X Y Z$ stage at the beamline (shown in Figure 4a) using the kinematic mounts.

1. Take care to avoid pulling the stages along their direction of travel. The magnets in the kinematic mounts are quite strong so this can be done quite easily by accident.

2. When approaching the mount, the chip holder should be held at a slight angle $\left( \pm 30^{\circ}\right)$. When the magnets make contact allow the chip holder to rotate parallel to the floor $\left(0^{\circ}\right)$ and the chip holder will click into place (Figure 4b).

3. When unloading a chip follow a reverse path. Rotate and angle the chip away from the stages before pulling the chip holder away.

2. Using the beamline's on-axis viewing system and the chip alignment GUI, locate the top left fiducial of the chip. Fiducials are three squares, two small and one large, at right angles to one another (Figure 5a). The chip is back illuminated so the chip will appear dark with apertures as white squares.

3. Center on fiducial zero in $X, Y$, and $Z$ (Figure 5b). Align $X$ and $Y$ by moving left/right and up/down, respectively. Align $Z$ by moving the chip in and out of focus.

\section{Click Set Fiducial Zero.}

5. Repeat step 3.2 for fiducial one (top right, Figure $5 \mathbf{c}$ ) and fiducial two (bottom left, Figure 5d) to align all fiducials with the X-ray beam.

6. Generate a co-ordinate matrix by pressing make coordinate system, this calculates the offset, pitch, roll, and yaw of the chip relative to the stages allowing all subsequent movements to be done in the chip coordinate frame.

7. Click Block Check to move the XYZ stage to the first well of each city block for visual confirmation that the chip is well aligned.

8. If the X-ray crosshair lines up with the apertures the chip is aligned. If not, repeat steps 3.2-3.3.

NOTES: In case of difficulty aligning (broken fiducials), different apertures on the chip can be used for alignment using the "alignment type" pull-down menu. Many different types of chip are available for fixed target 
data collection. Different chip types are accommodated through use of the 'chip type' pull-down menu. The most common chip types used at I24 are 'Oxford' and 'custom' chips. The number and the spacing of apertures and fiducials on the chip are read from a chip dictionary defined via the pull-down menu. Custom chip allows the aperture spacing to be defined on-the-fly, which is particularly useful for thin-film sheet-on-sheet or other 'chipless' type chips where crystals are randomly located across the holder ${ }^{37}$. A new Python GUI, offering moveon-click functionality and automated chip alignment is currently under development, but is not yet ready for routine use at the time of the writing of this manuscript.

\section{Setting up data collection}

NOTE: Data collection setup will depend on the system being studied, and the experiment to be performed. This can range from the simplest SSX experiment, collecting a low dose structure, to a time-resolved experiment using lasers or rapid mixing to initiate a reaction which will require multiple complete datasets at different time delays. To set up a data collection the following parameters need to be defined.

1. Experimental variables: Fill in the folder, filename, exposure time, transmission, detector distance, and number of shots per aperture in as appropriate.

2. Chip type: As described above, match the chip type to the chip in use.

1. If a thin film or 'chipless' chip is being used, then set the chip type to None.

2. Define the number of steps and step size in both $x$ and $y$ in the GUI.

3. Set the map type: this allows subsections of a chip to be selected for data collection (Figure 3b). 'None' means data are collected from every aperture on a chip. 'Lite' means data are collected from selected city blocks on the chip (Figure 3b). This can be useful if, for example, a region of a chip is known to be poorly loaded or empty. 'Full' allows individual apertures to be selected for data collection. In this case a correctly formatted text file must be provided. Details and a template can be obtained from beamline staff.

4. Pump-probe: Select the type of pump probe experiment and the desired time delay. The triggering of the pump (usually a LED or laser) is often specific to a particular experiment, so will not be described in detail here.

1. 'Short' delays refer to experiments when there is a dwell at each aperture between the pump and the probe (i.e., pump, probe, 'move to the next sample.) Delays are typically on the order of 1 second or tens of milliseconds.

2. Long' delays refer to an excite and visit again (EAVA) strategy, where apertures are visited twice, with a defined time delay between visits (i.e., pump, move, pump, move, probe, move, probe, etc.). The time delay is calculated and $\mathrm{X}$-ray exposure times (Figure 3c) and it is typically $\sim 1$ second or more.

\section{Common data collection methods}

NOTE: The following are the key parameters that define the type of experiment being carried out. This section assumes that the other settings from protocol 3 "Setting up Data Collection" have been defined.

1. Scenario 1: Low-dose data collection. Collection of a single diffraction image from every selected aperture on the sample holder.

1. Set number of shots per aperture to 1 . 
2. Set pump probe to None.

2. Scenario 2: A dose series, collecting $n$ images sequentially from every selected aperture on the sample holder. The chip is stationary at each aperture while each set of $n$ images is collected.

1. Set the number of shots per aperture to ' $n$ '. Note that processing is simplified if $n=5,10,20$ or another multiple of 10. It is difficult to establish trends if $n<$ 5. It is useful to consider the total time required to cover a chip and the number of image files produced when $n$ is increased.

2. Set pump probe to None.

3. Scenario 3: Pump-probe methods

1. Select a method from the Pump Probe pull-down menu to open the Laser Excitation Control Centre.

2. For a pump probe experiment fill in the Laser Dwell at each aperture option.

3. For EAVA fill in the Laser Dwell at each aperture and X-ray exposure and click Calculate.

4. Select the appropriate Repeat option in the edm GUI pump probe drop-down menu for the desired delay time.

5. If the experiment requires a pre-illumination step fill in the Laser 2 Dwell section.

6. After all experimental variables are defined press Set parameters and create short list. This loads experimental variables onto the geobrick controller. After this is done pressing Start will move the detector in, the backlight out, and start data collection. At all points in setting up data collection it is useful to have a terminal window open where feedback on the status and outcome of each of the steps is printed.

\section{Data processing}

NOTE: Broadly speaking data processing can be divided into three groups based on the urgency with which feedback is required. Fast feedback is required to show if crystals are present and diffract, and if so, in what numbers. This should keep up with data collection. Performing data indexing and integration which can be slower but should still be performed on comparable time scales with data collection. Merging and scaling of reflection intensities into an mtz file for structure solution and the generation of electron density maps represents the final step and can be slower still. Here starting pipelines at I24 for the first two stages only will be discussed, as they are required for real-time feedback to guide your experiment, though note that metrics such as hit-rates and scaling statistics are not a substitute for inspecting electron density, which may provide the only confirmation that a ligand has bound, or a reaction occurred, in crystallo.

1. Fast feedback

1. To load the data processing modules type module load i24-ssx into the terminal on any beamline workstation.

2. To run the hit-finding analysis type i24-ssx /path/ to/visit/directory/ into the terminal: i24-ssx /dls/i24/ data/2020/mx12345-6/

NOTE: This opens three terminal windows and, once data has been written to disk, a graphical representation of spot finding results from Diffraction Integration for Advanced Light Sources (DIALS) 40,41 (Figure 6a). 
1. Default settings scores every $10^{\text {th }}$ image and refreshes every few seconds to minimise the computational load.

2. Change the default by adding an argument to the end of the command above. For example, 'i24-ssx /dls/i24/data/2020/mx12345-6 2' i24ssx would run hit finding on every other image. However, this can put undue strain on the cluster (a shared resource!) and slow down processing times. The graph is color coded based on the likelihood of successful indexing, red shows at least 15 Bragg spots have been found (good chance of indexing), blue shows little to no useful diffraction.

3. View diffraction images of interest in the DIALS image viewer by clicking on the spots on the spot finder interface.

2. Indexing and integration feedback

NOTE: Indexing and integration of diffraction data are performed with DIALS using the dials.still_process function 40,41 . As such, specific information relating to your crystal (expected crystal space group, unit cell, and an experiment geometry) should be put into a .phil text file.

1. Load DIALS modules by typing module load dials in a terminal.

2. To begin processing a dataset type dials.still_process /path/to/images//pathto/philfile.phil. The progress of all still processing datasets can be monitored by running the stills_monitor script by typing monitor_stills_process.py (after performing module load i24-ssx and changing directory to the current visit) (Figure 6b).
3. The unit cell distribution of indexed diffraction data (Figure 7a) can be monitored using the command ctbx.xfel.plot_uc_cloud_from_experiments/ path/to/dials/output/*refined.expt combine_all_input=true This is particularly useful to identify and resolve unit cell polymorphs as discussed previously 42 .

4. 'Visualzie' if, and how, this distribution varies across a fixed target by producing a 2D plot (Figure 7b) using the command python pacman.py /visit/ processing/_hit_finding/chip.out.

5. Produce stereographic projections of all indexed diffraction data (Figure 7c) using the DIALS command dials.stereographic_projection hkl= 0,0,1 expand_to_P $1=$ True /path/to/dials/output $/$ *refined.expt.

NOTE: It is a common pathology when processing stills data from crystals where the symmetry of the Bravais lattice is higher than the space group symmetry that merged data appear as a perfect twin. Data processing algorithms have evolved to resolve this pathology $43,44,45,46$ but users should be mindful of this while processing their data.

\section{Representative Results}

\section{Low Dose Data Collection and Series}

Low dose (Step 5.1: Scenario 1) and dose series (Step 5.2: Scenario 2) data were collected on copper nitrite reductase micro crystals at I24 and have been published previously 42 . All samples were prepared as described in step 1, data collected as per steps 3,4 , and 5 , and processed using methods in step 6. In this work a rapid dose series was collected with 20 diffraction images taken at each aperture 
(i.e., $n=20$ in the data collection GUI shown above) before moving to a fresh sample. From these data a bimodal distribution of unit cells in space group P213 was identified $(a=b=c=97.25 \AA$, and $a=b=c=96.38 \AA)$. Identifying and separating these unit-cell polymorphs for processing showed a marked improvement in data quality indicators and revealed two different structures in a flexible loop between residues 189-193 instead of the mixed state observed when processing all data together. Identification of such polymorphs could make all the difference in a delicate time-resolved structural study where only small structural changes are expected. Furthermore, the dose series collected revealed a dose dependent unit cell change in the crystal, with increased dose shifting the population in favor of the larger unit-cell.

Similar work was performed by Ebrahim et al $(2019)^{47}$, where a dose series (Step 5.2: Scenario 2) was collected from a dyetype heme peroxidase from Streptomyces lividans (DtpAa) to compare low dose structures from SSX (Step 5.1: Scenario 1) with those measured in the same fixed target system using SFX. SFX data were collected at SACLA Beamline BL2 EH3 with a pulse length of 10 femtoseconds and a repetition rate of $30 \mathrm{~Hz}$. The 10 femtosecond pulse duration ensures that dose dependent effects are not present in the SFX data. SFX data were compared to SSX data collected on beamline 124 , where 10 sequential 10 millisecond exposures were measured at each sample position (i.e., $n=10$ ). The dose dependent migration of a heme iron coordinated water molecule away from the iron was observed, as well as a conformational change in one of the heme propionate groups in the SSX dose series. Although not damage-free like the SFX structure, the dose series allowed the Fe-O bond length of a zero-dose dataset (ferric heme) to be extrapolated, with this agreeing within experimental error with the value obtained from SFX.

The serial crystallography data collection methods described here can also be easily adapted to provide new sample environments to, for example, study anaerobic protein structures at room temperature. As outlined in Rabe et al $2020^{48}$, loading a 'sheet-on-sheet' sample, or 'chipless chip', with different sealing films in an anerobic chamber enables room temperature collection of structural data from dioxygensensitive samples.

\section{Pump Probe}

Although the following representative results were not collected at Diamond Beamline 124, these methods have been developed in close collaboration between facilities in the iNEXT program to work towards standard methods in serial crystallography method development. Beamline I24 offers, or will soon offer, equivalent collection methods to those described below to perform such experiments using the methods described in the protocols above.

\section{Pump Probe: Rapid Mixing}

Rapid mixing SSX has been performed at beamline TREXX at PETRA III by Mehrabi et al (2019) ${ }^{28}$ using a piezo driven droplet injector to initiate reactions on fixed targets. This work presents a proof of principle on chip mixing experiment binding GlcNac3to lysozyme microcrystals, with binding occurring within $50 \mathrm{~ms}$ of a $75 \mathrm{pL}$ drop being applied to the sample. This study was followed up with a 7structure time-resolved series of xylose isomerase activity, demonstrating glucose binding within $15 \mathrm{~ms}$ and the formation of an open ring conformation in the glucose molecule after a 60 second time delay. An equivalent setup for droplet injection is currently under development for use on 124 . 


\section{Pump-Probe: Light Activation}

A light activated pump-probe serial experiment is presented in Schulz et al (2018) ${ }^{49}$. Fluoroacetate dehydrogenase was soaked with photocaged fluoroacetate and pumped with $320-360 \mathrm{~nm}$ laser light to produce structures at 4 time points $(\mathrm{t}=0,30,752$, and $2,052 \mathrm{~ms})$. The resting state structure (0 ms) shows an empty active site, with the exception of a few water molecules, and equivalent density between the cap domains of both protein subunits. $30 \mathrm{~ms}$ and 752 ms after light activation a significant reduction in electron density can be observed in the cap domain of subunit B relative to subunit $A$. The reduction in electron density in the cap domain of subunit B coincides with the appearance of fluoroacetate in the active site of subunit $A$ at $752 \mathrm{~ms}$. The final dataset at 2,052 ms shows further structural rearrangement of the ligand, suspected to facilitate the correct geometry for $\mathrm{S}_{\mathrm{N} 2}$ attack, and potential formation of an intermediate state in the reaction. On I24, a portable Pharos laser system which is tunable from $210-2500 \mathrm{~nm}$ providing femtosecond pulses can be used for light-activation. Initial experiments showed the successful activation of a photocage using 308 $\mathrm{nm}$ excitation with binding of the released ligand to the target protein observed. At the time of writing integration into the beamline personnel safety system is ongoing and routine user experiments are anticipated in early 2021. For experiments when less intense pulses of light are required, light-activation with TTL controlled LEDs has been performed successfully. 


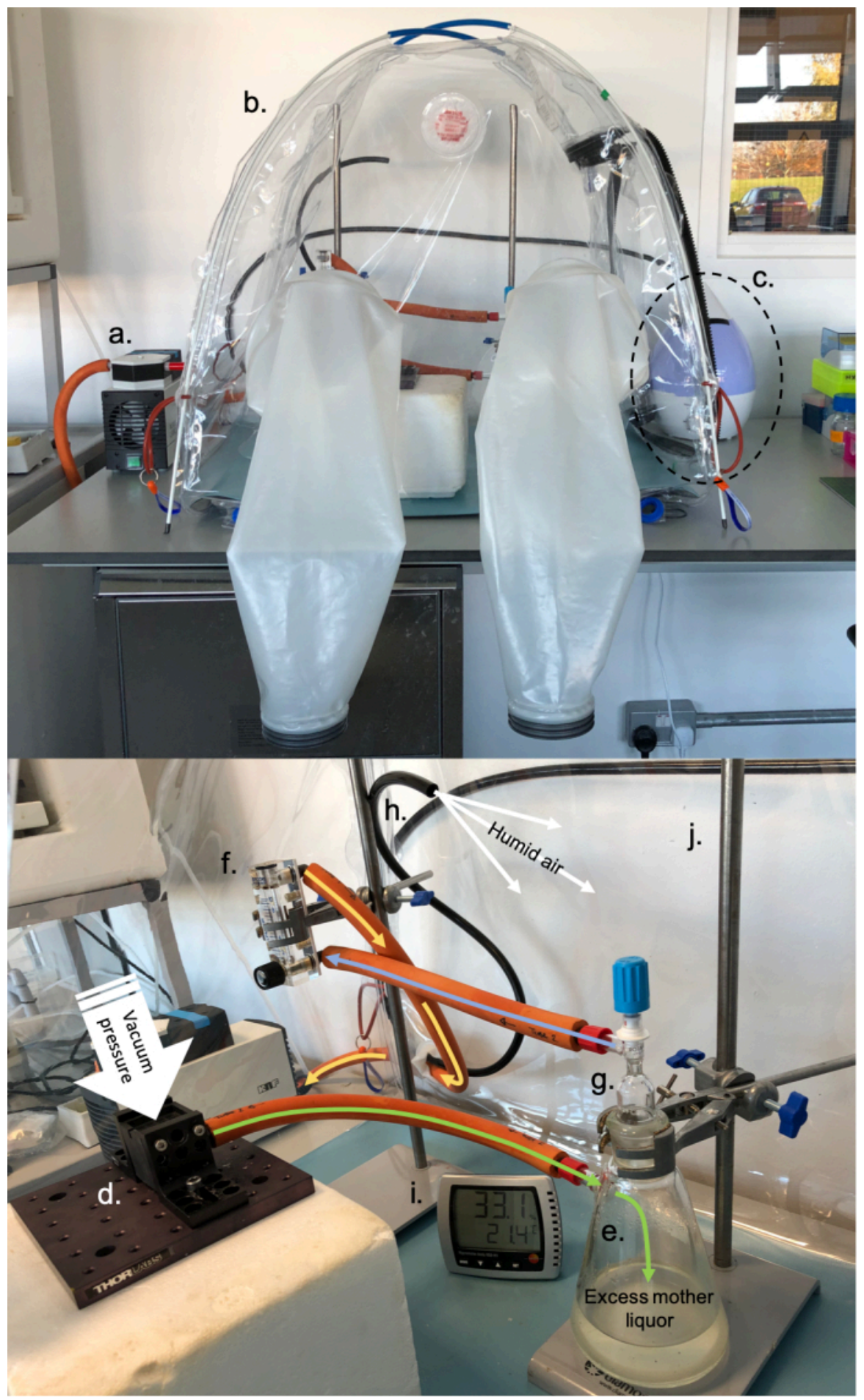

Figure 1: Sample loading equipment in place at Diamond Light Source. The set-up consists of a vacuum pump (a), glove-box (b), and humidifier (c). Within the glove-box vacuum pressure is used to act on a chip loaded with crystal slurry held in a sample block (d) attached to a Buchner flask (e, green arrow), via a pressure regulator (f, yellow arrow) attached to a stopcock (g, blue arrow). Humid air is pumped into the tent via plastic tubing attached to the humidifier (h), and measured 


\section{jove}

using a hygrometer (i). Components are held in place using clamp stands (j). Please click here to view a larger version of this figure.

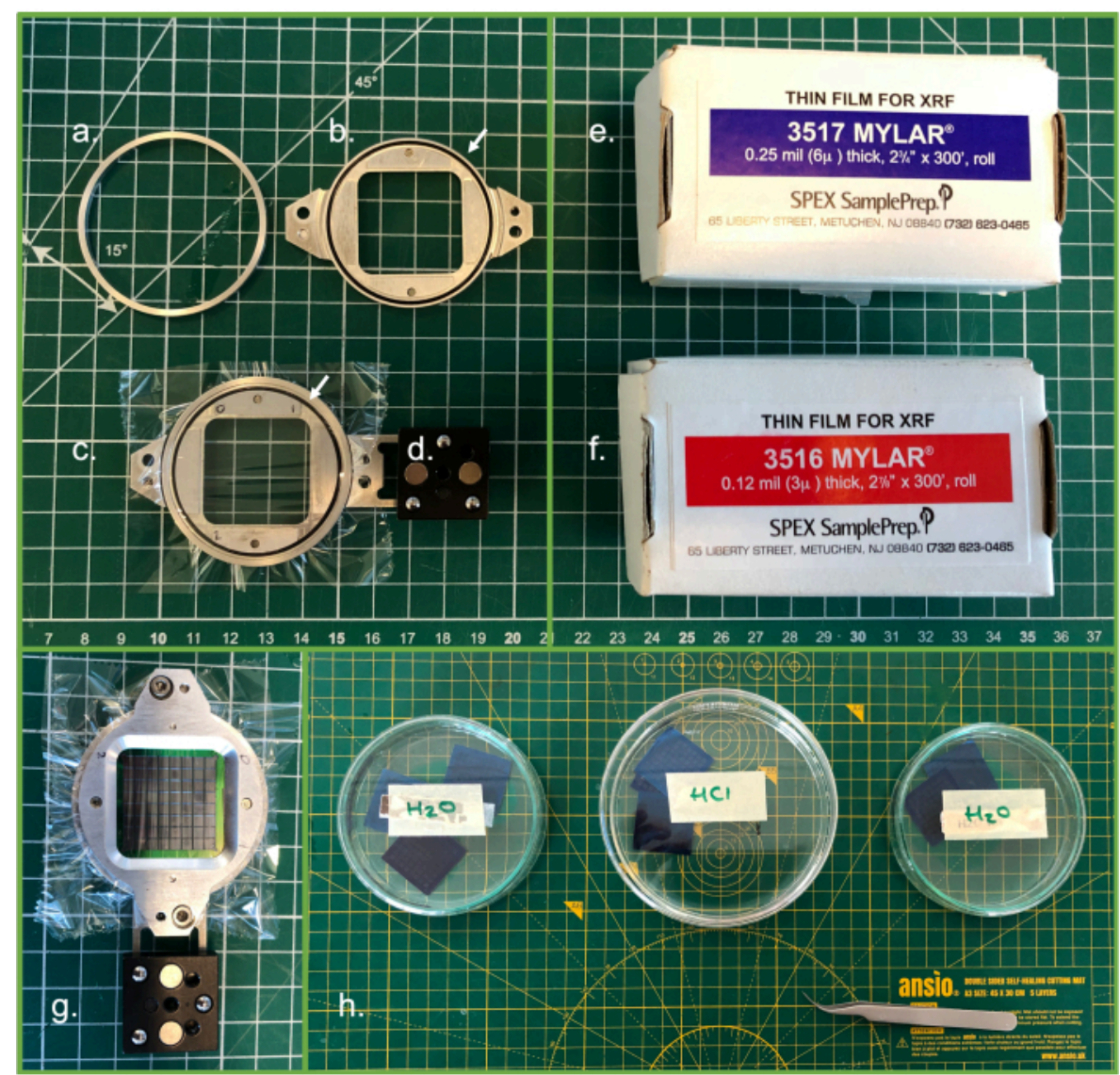

Figure 2: Sample holders. They utilize a metal O-ring (a) to clamp polyester film onto a top (b) and bottom (c) half, with the bottom half sporting magnetic mounts (d) that are used to attach the sample holder to the sample stages. The polyester film (6 $\mu \mathrm{m}(\mathbf{e})$ or $3 \mu \mathrm{m}$ (f)) as well as rubber O-rings (white arrows) prevent a crystal-loaded chip from drying rapidly in a sample holder which is closed tight with hex bolts $(\mathbf{g})$. Chips are cleaned using sequential 15-minute baths in $\mathrm{dH}_{2} \mathrm{O}, 1 \mathrm{M} \mathrm{HCl}$, and $\mathrm{dH}_{2} \mathrm{O}(\mathbf{h})$. Please click here to view a larger version of this figure. 


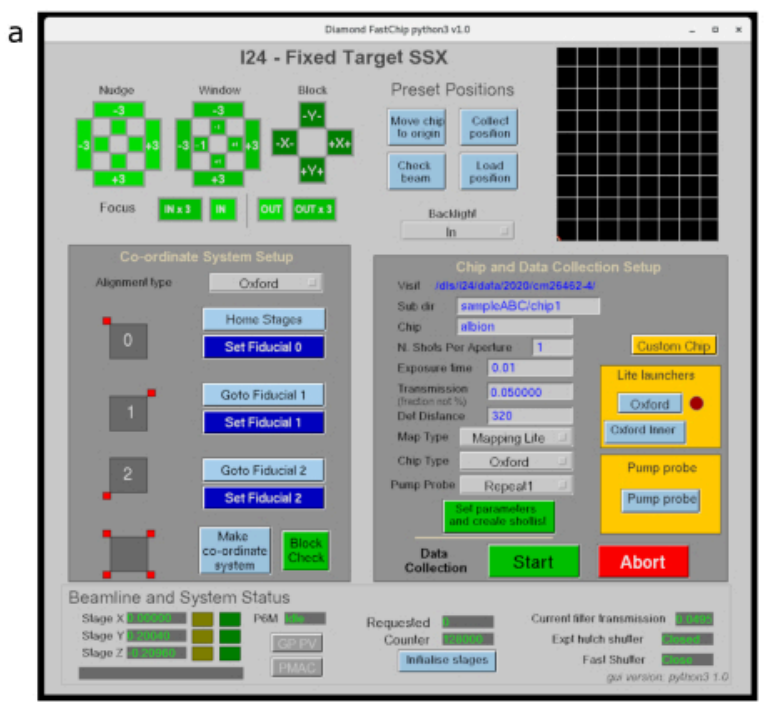

b

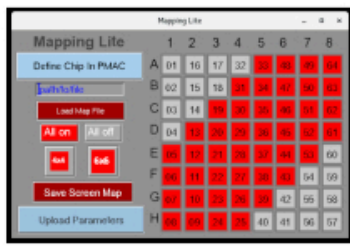

c

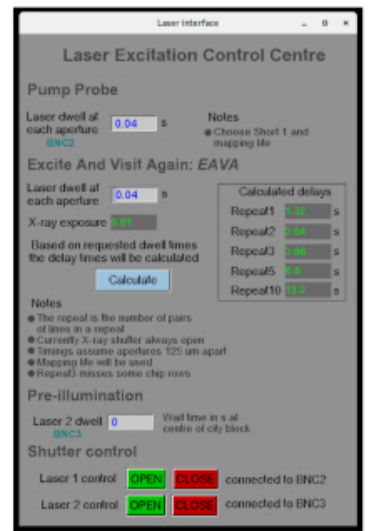

Figure 3: Data collection GUI for fixed target data collection at I24. (a) Shows the main interface used for aligning chips and defining data collection parameters, (b) is the mapping lite interface used for defining sub-regions of a chip for data collection and (c) is an interface for defining parameters for laser illumination. Please click here to view a larger version of this figure. 


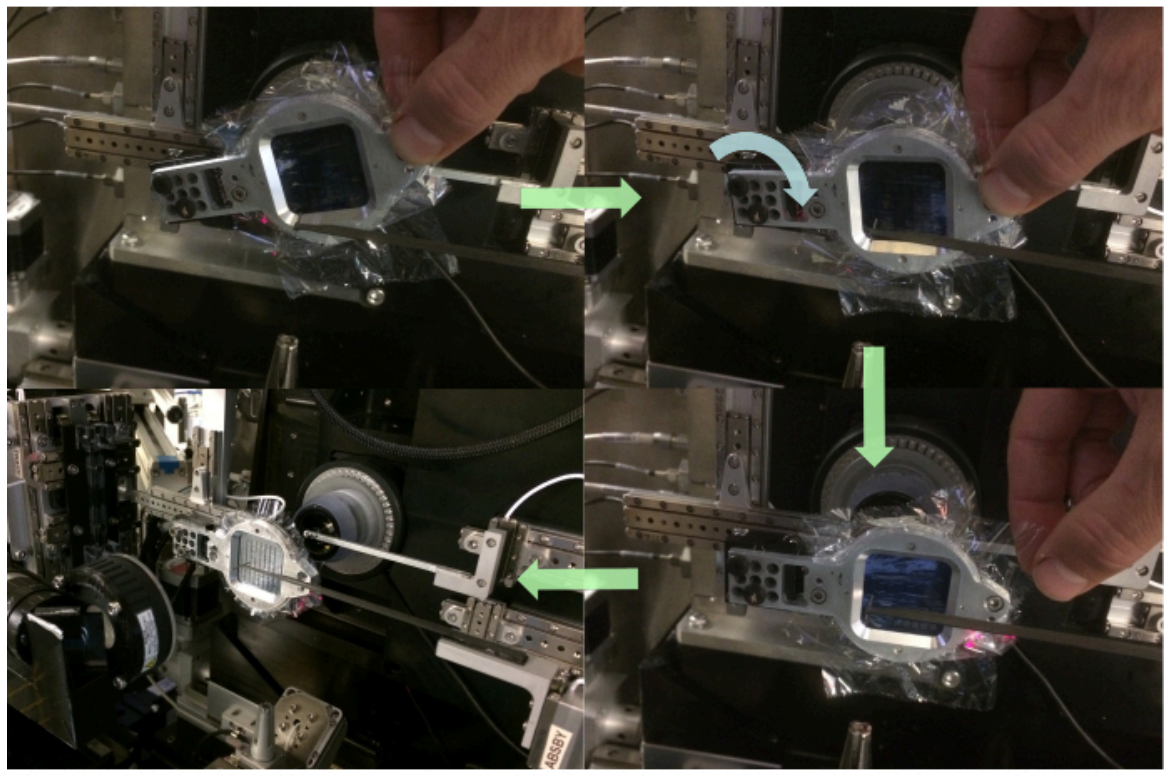

Figure 4: The process of mounting a chip holder onto the stages as described in Step 3, point 1. Please click here to view a larger version of this figure. 
a
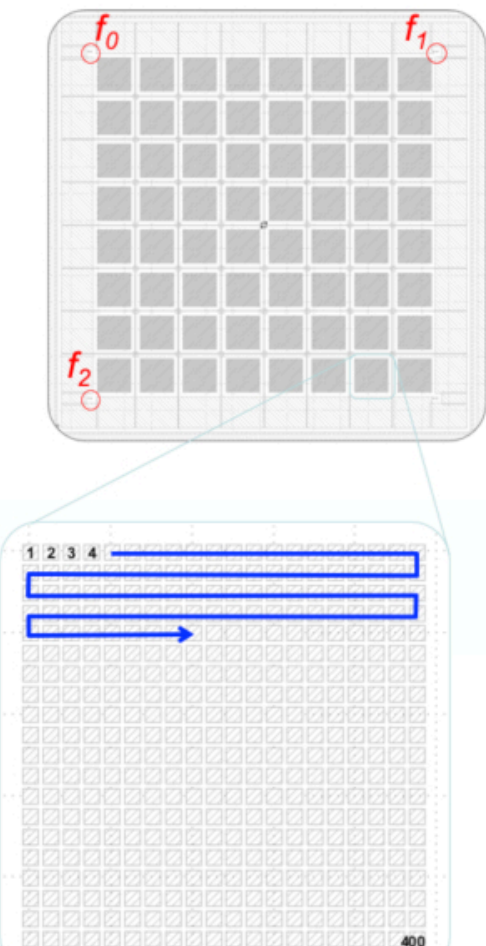

Figure 5: Chip alignment. A chip is aligned by clicking on three fiducial markers on the chip shown in (a). Views of fiducials 0,1 and 2 through the beamline on-axis viewing system are shown in (b), (c) and (d). Please click here to view a larger version of this figure. 
a
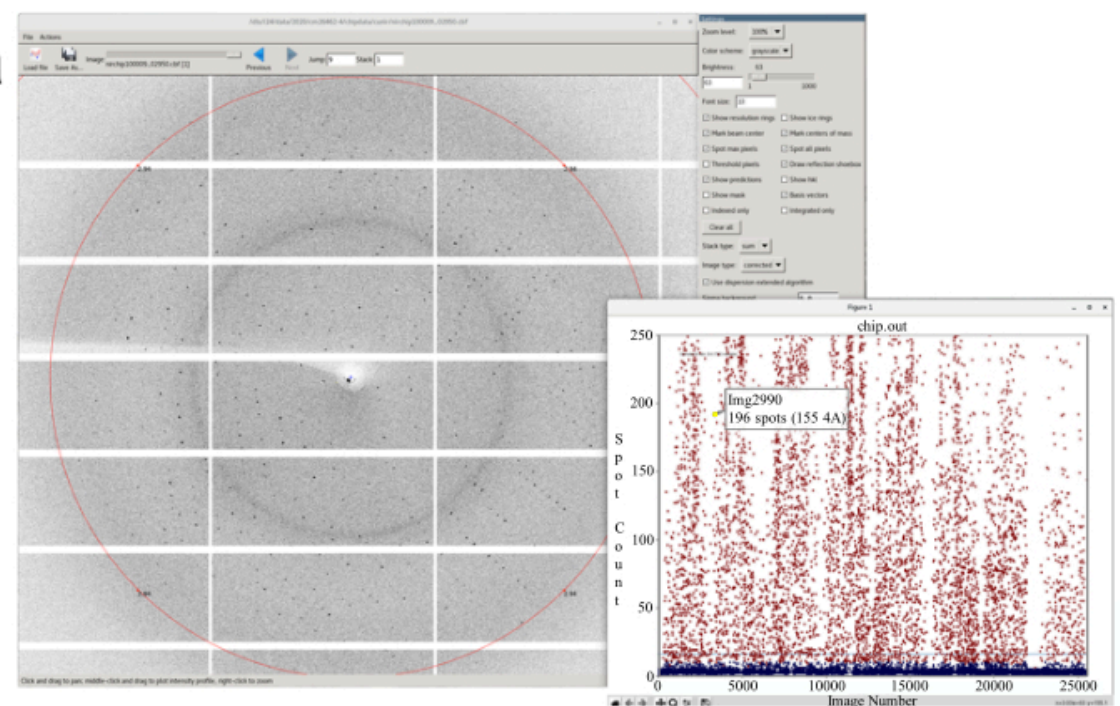

b

\begin{tabular}{|c|c|c|c|c|c|c|}
\hline Name & Processed & Indexed & $\%$ & Integ rated & $\%$ & \\
\hline atomic & 25600 & 10737 & $16 \%$ & 14025 & $55 \%$ & \\
\hline busted_JR & 25600 & 11009 & $43 \%$ & 14698 & $57 \%$ & \\
\hline cheeky & 3500 & 1520 & $43 \%$ & 2628 & $75 \%$ & \\
\hline dakota_JR & 25600 & 6049 & $24 \%$ & 7710 & $30 \%$ & \\
\hline europe_JR & 22200 & 958 & $4 \%$ & 1040 & $5 \%$ & \\
\hline fergie_JR & 25600 & 10142 & $40 \%$ & 13247 & $52 \%$ & \\
\hline goldie_JR & 25600 & 9153 & $36 \%$ & 11639 & $45 \%$ & \\
\hline hardfi_JR & 25600 & 7494 & $29 \%$ & 9166 & $36 \%$ & \\
\hline jarule & 25600 & 9418 & $37 \%$ & 12398 & $48 \%$ & \\
\hline mansun & 25600 & 10211 & $40 \%$ & 15908 & $62 \%$ & \\
\hline mansun_JR & 25600 & 10692 & $42 \%$ & 17943 & $70 \%$ & \\
\hline nolans & 25600 & 7470 & $29 \%$ & 11097 & $43 \%$ & \\
\hline nolans_JR & 25600 & 7689 & $30 \%$ & 11932 & $47 \%$ & \\
\hline others & 25600 & 5000 & $20 \%$ & 6978 & $27 \%$ & \\
\hline pogues & 5480 & 2445 & $45 \%$ & 3539 & $65 \%$ & 1 \\
\hline quincy & 8636 & 4155 & $48 \%$ & 5715 & $66 \%$ & I \\
\hline rascal & 7351 & 4127 & $56 \%$ & 5845 & $80 \%$ & / \\
\hline smiths & 801 & 268 & $33 \%$ & 359 & $45 \%$ & - \\
\hline travis & 16315 & 10061 & $62 \%$ & 14771 & $91 \%$ & 1 \\
\hline
\end{tabular}

Figure 6: Auto-processing results displays launched as described in step 6.1. An updating hit-rate plot is displayed (a, inset). If a 'hit' is clicked on the corresponding diffraction image is displayed in dials image viewer. The hit-rate for the current data collection is shown (29.6\% in this example). Panel (b) shows an example of a window showing current indexing and integration rates for data collected so far during the visit that updates in real time. Please click here to view a larger version of this figure. 

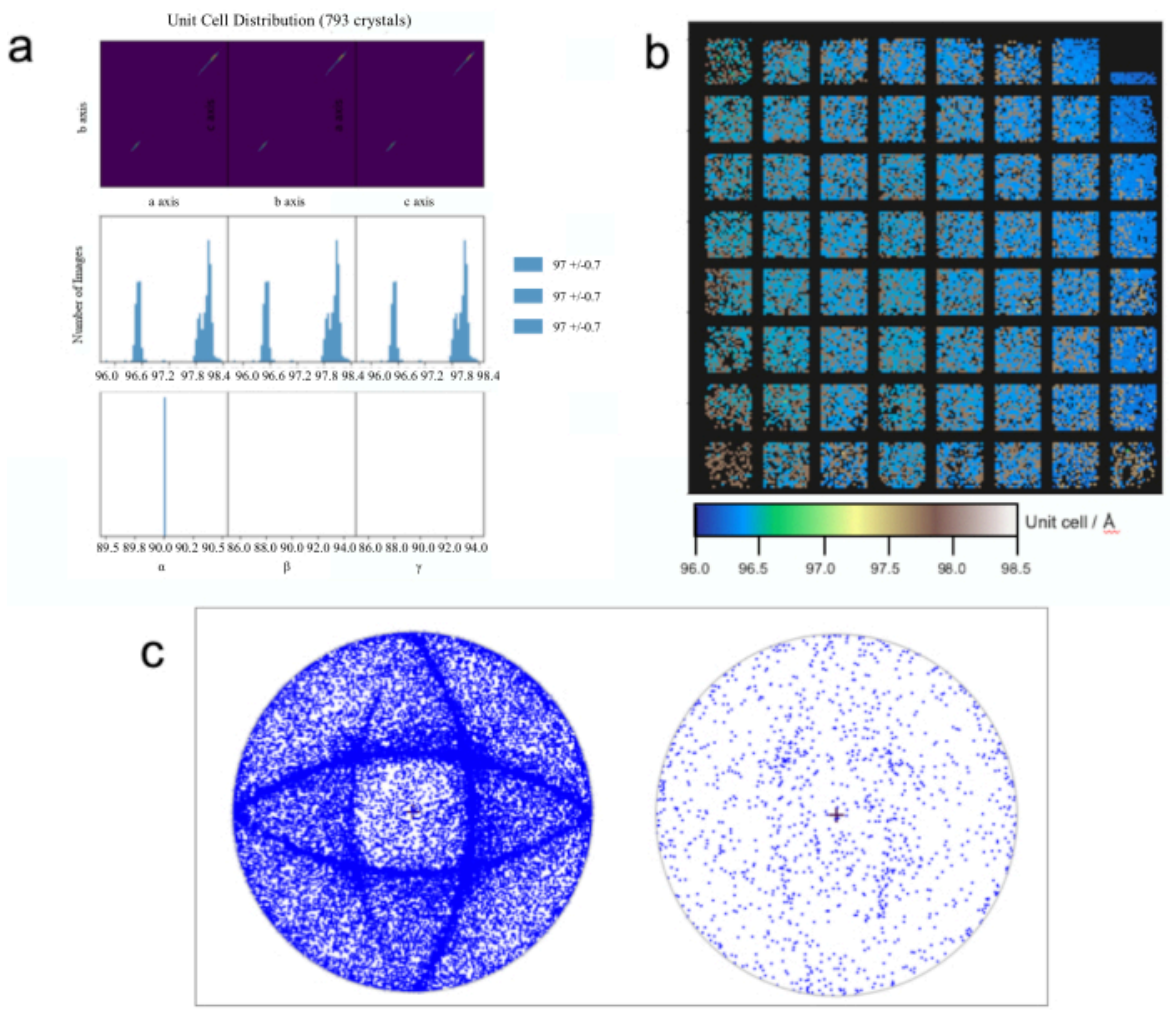

Figure 7: More in-depth data analysis. Visualization of unit cell parameters can reveal polymorphs (a). Average unit cell parameters are calculated; however, this does not yet extend to individual averages for polymorphs. Visualization of a small subset of data (data shown are a subset of 793 copper nitrite reductase crystals from the data described in Ebrahim et al 2019) is often sufficient to reveal trends. 2-D plots of useful parameters can also be produced to reveal variations that arise due to loading or dehydration effects that could be addressed for upcoming data collections (b). Stereographic projections can reveal the presence, or absence, preferred orientations feeding back into the loading protocol (c). Please click here to view a larger version of this figure.

\section{Discussion}

Serial synchrotron data collection is a relatively new technique at MX beamlines, bridging the gap between the ultra-fast data collections currently being performed at XFELs and traditional synchrotron-based MX. This manuscript aims to give an overview of how to successfully collect fixed target serial data at beamline 124, Diamond Light Source for low dose, dose series, and time-resolved experiments. As with standard crystallography, sample preparation is a major bottle neck in structure solution. SSX is no different, and preparation of a homogenous crystal slurry in sufficient quantities has not yet benefited from several decades of study and refinement like the growth of single large protein crystals has. However, preparation of these slurries is outside the scope of this paper and has been summarized elsewhere ${ }^{36}$. The critical step in the approach described here involves the careful use of the available sample using easy to use GUI interfaces (step 3) 
and automated data processing pipelines (step 6) to inform the chip loading (step 1) and how an experiment should proceed.

The fast feedback pipeline is a powerful tool that allows users to assess initial hit rates during data collection to inform subsequent chip loading protocols for successful data collection. When faced with a low hit rate $(<5 \%)$, users risk collecting incomplete data and/or wasting beamtime with additional collections. In this case, sample could be pooled, concentrated by gentle centrifugation, and/or larger volumes could be loaded in step 1.5. A higher hit rate is generally favorable, however, there is a point of diminishing return where overloading leads to multiple crystals in the same well. DIALS is capable of dealing with multi-lattice diffraction data $^{50}$, but a greater concern than indexing and integration is the detrimental effect crystal grouping can have on the even activation of crystals by laser light or rapid mixing for precise time resolved experiments. Particular care should therefore be taken to avoid overloading fixed targets for time resolved experiments.

The indexing and integration processing step produces a plot with the central cross representing the beam direction, each point representing the direction of the hkl 001 reflection of individual lattices, and the outer ring of the circle representing a rotation of $90^{\circ}$ away from the beam axis. This will show if your crystals have a preferred orientation, which may impact data completeness and indicate the need to collect more data or vary the loading protocol. In the left-hand panel of Figure 7c, the effect of overloading a chip with HEWL crystals is shown. As apertures fill with more crystals, they stick to the angled walls of the apertures rather than wedging at the base in a random orientation. The two orthogonal ellipses are a result of crystals lying on the internal walls of the chip which are at $\sim 35^{\circ}$ to the beam direction. This reduces the volume of crystals loaded, reduces the hit rate, and dramatically reduces the fraction of crystals lying in these preferred planes.

It should be noted that other serial approaches are available at 124 , such as LCP extruders and microfluidic chips. These use similar GUls and the same processing pipelines so much of the above will remain applicable even if a different technique is used. A number of serial approaches exist for both SSX and SFX beyond the fixed target approach described here, each has certain advantages over the other depending on the experiment to be performed and the beamline used for the experiment. As serial approaches are evolving rapidly it is advisable to check the beamline webpages (https://www.diamond.ac.uk/ Instruments/Mx/l24.html) for recent updates and talk to beamline staff at as early a stage as possible when planning beamtime. Access to I24 for standard and serial experiments is free at point of use. For UK and EU users travel and accommodation costs are partly covered through iNEXT Discovery.

\section{References}

1. Schlichting, I., Serial femtosecond crystallography: the first five years. IUCrJ. 2 (2), 246-255 (2015).

2. Diederichs, K., Wang, M., Serial Synchrotron XRay Crystallography (SSX). In Protein Crystallography: Methods and Protocols. Wlodawer, A., Dauter, Z., Jaskolski, M., Eds. Springer New York: New York, NY, 239-272 (2017).

3. Pearson, A. R., Mehrabi, P., Serial synchrotron crystallography for time-resolved structural biology. Current Opinion in Structural Biology. 65, 168-174 (2020). 
4. Chapman, H. N., Structure Determination Using X-Ray Free-Electron Laser Pulses. In Protein Crystallography: Methods and Protocols. Wlodawer, A., Dauter, Z., Jaskolski, M., Eds. Springer New York: New York, NY, 295-324 (2017).

5. Chavas, L. M., Gumprecht, L., Chapman, H. N., Possibilities for serial femtosecond crystallography sample delivery at future light sources. Structural Dynamics. 2 (4), 041709 (2015).

6. Dauter, Z., Wlodawer, A., Progress in protein crystallography. Protein \& Peptide Letters. 23 (3), 201-10 (2016).

7. Owen, R. L., Rudiño-Piñera, E., Garman, E. F., Experimental determination of the radiation dose limit for cryocooled protein crystals. Proceedings of the National Academy of Sciences of the United States of America. 103 (13), $4912-7$ (2006).

8. Garman, E. F., Weik, M., X-ray radiation damage to biological samples: recent progress. Journal of Synchrotron Radiation. 26 (Pt 4), 907-911 (2019).

9. Axford, D. et al. In situ macromolecular crystallography using microbeams. Acta crystallographica. Section D, Biological crystallography. 68 (Pt 5), 592-600 (2012).

10. Warren, A. J., Axford, D., Paterson, N. G., Owen, R. L., Exploiting Microbeams for Membrane Protein Structure Determination. Advances in Experimental Medicine and Biology. 922, 105-117 (2016).

11. Sanishvili, R., Fischetti, R. F., Applications of XRay Micro-Beam for Data Collection. In Protein Crystallography: Methods and Protocols. Wlodawer, A., Dauter, Z., Jaskolski, M., Eds. Springer New York: New York, NY, 219-238 (2017).
12. Wierman, J. L. et al. Fixed-target serial oscillation crystallography at room temperature. IUCrJ. 6 (2), 305-316 (2019).

13. Maeki, M. et al. Room-temperature crystallography using a microfluidic protein crystal array device and its application to protein-ligand complex structure analysis. Chemical Science. 11 (34), 9072-9087 (2020).

14. Grunbein, M. L., Nass Kovacs, G., Sample delivery for serial crystallography at free-electron lasers and synchrotrons. Acta Crystallographica Section D. 75 (2), 178-191 (2019).

15. Weierstall, U., Liquid sample delivery techniques for serial femtosecond crystallography. Philosophical Transactions of the Royal Society B: Biological Sciences. 369 (1647), 20130337 (2014).

16. Botha, S. et al. Room-temperature serial crystallography at synchrotron X-ray sources using slowly flowing free-standing high-viscosity microstreams. Acta crystallographica. Section D, Biological crystallography. 71 (Pt 2), 387-97 (2015).

17. Kovácsová, G. et al. Viscous hydrophilic injection matrices for serial crystallography. IUCrJ. 4 (Pt 4), $400-410$ (2017).

18. Monteiro, D. C. F. et al. A microfluidic flow-focusing device for low sample consumption serial synchrotron crystallography experiments in liquid flow. Journal of Synchrotron Radiation. 26 (2), 406-412 (2019).

19. Monteiro, D. C. F. et al. 3D-MiXD: 3D-printed Xray-compatible microfluidic devices for rapid, lowconsumption serial synchrotron crystallography data collection in flow. IUCrJ. 7 (Pt 2), 207-219 (2020). 
20. Mueller, C. et al. Fixed target matrix for femtosecond time-resolved and in situ serial micro-crystallography. Structural Dynamics. 2 (5), 054302 (2015).

21. Owen, R. L. et al. Low-dose fixed-target serial synchrotron crystallography. Acta Crystallographica Section D: Structural Biology. 73 (Pt 4), 373-378 (2017).

22. Keedy, D. A. et al. Mapping the conformational landscape of a dynamic enzyme by multitemperature and XFEL crystallography. eLife. 4 (2015).

23. de la Mora, E. et al. Radiation damage and dose limits in serial synchrotron crystallography at cryo- and room temperatures. Proceedings of the National Academy of Sciences. 117 (8), 4142-4151 (2020).

24. Barends, T. R. et al. Direct observation of ultrafast collective motions in $\mathrm{CO}$ myoglobin upon ligand dissociation. Science. 350 (6259), 445-50 (2015).

25. Pande, K. et al. Femtosecond structural dynamics drives the trans/cis isomerization in photoactive yellow protein. Science. 352 (6286), 725-9 (2016).

26. Standfuss, J., Spence, J., Serial crystallography at synchrotrons and X-ray lasers. IUCrJ. 4 (2), 100-101 (2017).

27. Grünbein, M. L. et al. Illumination guidelines for ultrafast pump-probe experiments by serial femtosecond crystallography. Nature Methods. 17 (7), 681-684 (2020).

28. Mehrabi, P. et al. Liquid application method for timeresolved analyses by serial synchrotron crystallography. Nature Methods. 16 (10), 979-982 (2019).

29. Beyerlein, K. R. et al. Mix-and-diffuse serial synchrotron crystallography. IUCrJ. 4 (Pt 6), 769-777 (2017).

30. Schmidt, M., Mix and Inject: Reaction Initiation by Diffusion for Time-Resolved Macromolecular
Crystallography. Advances in Condensed Matter Physics. 2013, 167276 (2013).

31. Kupitz, C. et al. Structural enzymology using X-ray free electron lasers. Structural Dynamics. 4 (4), 044003 (2017).

32. Stagno, J. R. et al. Structures of riboswitch RNA reaction states by mix-and-inject XFEL serial crystallography. Nature. 541 (7636), 242-246 (2017).

33. Shilova, A. et al. Current status and future opportunities for serial crystallography at MAX IV Laboratory. Journal of Synchrotron Radiation. 27 (5), 1095-1102 (2020).

34. Huang, C.-Y. et al. In meso in situ serial X-ray crystallography of soluble and membrane proteins. Acta Crystallographica Section D. 71 (6), 1238-1256 (2015).

35. Gao, Y. et al. High-speed raster-scanning synchrotron serial microcrystallography with a high-precision piezoscanner. Journal of Synchrotron Radiation. 25 (5), 1362-1370 (2018).

36. Beale, J. H. et al. Successful sample preparation for serial crystallography experiments. Journal of Applied Crystallography. 52 (Pt 6), 1385-1396 (2019).

37. Doak, R. B. et al. Crystallography on a chip - without the chip: sheet-on-sheet sandwich. Acta Crystallographica Section D: Structural Biology. 74 (Pt 10), 1000-1007 (2018).

38. Axford, D., Aller, P., Sanchez-Weatherby, J., Sandy, J., Applications of thin-film sandwich crystallization platforms. Acta Crystallographica Section F: Structural Biology Communications. 72 (Pt 4), 313-9 (2016).

39. Davy, B. et al. Reducing sample consumption for serial crystallography using acoustic drop ejection. Journal of Synchrotron Radiation. 26 (5), 1820-1825 (2019). 
40. Brewster, A. S. et al. Improving signal strength in serial crystallography with DIALS geometry refinement. Acta Crystallographica Section D. 74 (9), 877-894 (2018).

41. Winter, G. et al. DIALS: implementation and evaluation of a new integration package. Acta Crystallographica Section D. 74 (2), 85-97 (2018).

42. Ebrahim, A. et al. Resolving polymorphs and radiationdriven effects in microcrystals using fixed-target serial synchrotron crystallography. Acta Crystallographica Section D. 75 (2), 151-159 (2019).

43. Brehm, W., Diederichs, K., Breaking the indexing ambiguity in serial crystallography. Acta Crystallographica Section D. 70 (1), 101-109 (2014).

44. White, T., Processing serial crystallography data with CrystFEL: a step-by-step guide. Acta Crystallographica Section D. 75 (2), 219-233 (2019).

45. Shi, Y., Liu, H., EM-detwin: A Program for Resolving Indexing Ambiguity in Serial Crystallography Using the Expectation-Maximization Algorithm. Crystals. 10 (7), 588 (2020).

46. Gildea, R. J., Winter, G., Determination of Patterson group symmetry from sparse multi-crystal data sets in the presence of an indexing ambiguity. Acta Crystallographica Section D. 74 (5), 405-410 (2018).

47. Ebrahim, A. et al. Dose-resolved serial synchrotron and XFEL structures of radiation-sensitive metalloproteins. IUCrJ. 6 (4), 543-551 (2019).

48. Rabe, P. et al. Anaerobic fixed-target serial crystallography. IUCrJ. 7 (5), 901-912 (2020).

49. Schulz, E. C. et al. The hit-and-return system enables efficient time-resolved serial synchrotron crystallography. Nature Methods. 15 (11), 901-904 (2018).

50. Gildea, R. J. et al. New methods for indexing multilattice diffraction data. Acta Crystallographica Section D. 70 (10), 2652-2666 (2014). 